\title{
Autonomous Coastal Land Cover Assessment Using Polarimetric Decomposition of SAR Data
}

\author{
Bambang H. Trisasongko \\ Department of Soil Science and Land Resources and P4W/CRESTPENT, Bogor \\ Agricultural University. Jalan Meranti, Dramaga Campus. Bogor 16680. Indonesia. \\ Email: bambangtr@ipb.ac.id
}

\begin{abstract}
The paper reports an experiment on classification using fully polarimetric SAR data. Many reports have been presented mentioning test sites in temperate regions utilizing polarimetric SAR data from airborne and/or spaceborne SAR sensors. However, few studies are dedicated to tropical region which highly dynamic land uses are observed. Using the AirSAR Sungai Wain fully polarimetric data, capability to extract features in coastal region has been demonstrated by an unsupervised classification technique fed by the CloudePottier decomposition theorem.
\end{abstract}

Keywords: Cloude-Pottier Decomposition; Kalimantan; polarimetry; Sungai Wain; Synthetic Aperture Radar.

\section{Introduction}

Coastal is one of important ecosystems in the world, providing an interface between land and sea. Coastal mangrove area offers natural management of excess water and regulates flooding. The vegetation is also important to minimize impact of waves, including tsunami. Despite its importance, land use conversion in coastal areas has been witnessed around the world, including Indonesia.

Remote sensing and its applications on coastal region are thoroughly discussed, for example a review by Melack [1]. Due to immense data availability, optical images have been used both in research and operational basis. In three subtropical areas of North Africa, Ahmed, et al. [2] successfully implemented a series of Landsat data for coastal site characterization. In China, also using Landsat data, Huang, et al. [3] studied spatio-temporal change of a coastal area. These examples suggest that optical dataset has been useful to assist coastal zone monitoring.

However, in case of tropical region, cloud cover is one of the main challenges, which could lead to data gaps and improper analysis. Synthetic Aperture Radar (SAR) provides an alternative of data source which can be used individually or in conjunction with existing optical dataset through data fusion. SAR data have 
been assessed thoroughly, mostly using single or dual polarization data. Nonetheless very limited information has been presented on the use of fully polarized SAR data, specifically on tropical areas where cloud coverage has been an immense problem. With the availability of fully polarimetric data from currently operating satellites, further exploration of the data is therefore important to seek an efficient way in monitoring land cover of coastal region. The aim of this paper is to provide an assessment of those data for coastal land cover monitoring and to examine applicability of unsupervised classification of fully polarimetric data.

\section{Polarimetry and Polarimetric Decomposition}

Synthetic Aperture Radar (SAR) becomes a powerful sensor to monitor cloudpersistent areas in tropical region. SAR capability to capture data in virtually all-weather condition gains interests, including in Indonesia. However, SAR application is rather limited mostly due to the fact that recent systems provide data only in single polarization mode. For instance, ERS Advanced Microwave Imager has VV polarization, whereas Radarsat has $\mathrm{HH}$. Hence, difficulties arise and most data analyses are restricted to tone (in forms of amplitude or backscatter) and texture basis. Advanced breakthrough in sensor technology allows development of multi-polarization SAR systems within these decades.

One of the first implementations of multi-polarization SAR sensor has been initiated by NASA/Jet Propulsion Laboratory. AirSAR airborne system was designed to provide fully polarimetric SAR data. Earliest experiment from space was conducted by SIR-C/X-SAR mission and had provided spaceborne imaging for selected regions in the world. European Space Agency launched Envisat satellite, which also has polarimetric capability namely Advanced SAR. However, only 2 polarizations could be delivered to users. Fully polarimetric data from space are currently provided by Canada (Radarsat-2) and Japan (ALOS PALSAR).

One of advantages employing fully polarimetric SAR data is the possibility to discriminate scattering mechanisms from earth surface. A fully polarimetric SAR data can be represented by scattering matrix, usually defined as

$$
S=\left[\begin{array}{ll}
S_{h h} & S_{h v} \\
S_{v h} & S_{v v}
\end{array}\right]
$$

The scattering matrix is then analyzed with various approaches to retrieve information about scattering processes. Generally, the analysis is called target decomposition. Two groups of decompositions have been found in the 
literatures. The first is decomposition of complex voltage reflection (Sinclair) matrix. Two algorithms have been widely developed from this kind of matrix, i.e. Pauli decomposition and sphere/diplane/helix decomposition. The second approach is decomposition of power reflection (Müller/Kennaugh and covariance type) matrices. Further, the approach could be divided into two general techniques. The first is decompositions based on Müller/Kennaugh matrix (for instance Huynen decomposition). Others propose the use of covariance $[\mathrm{C}]$ or coherence $[\mathrm{T}]$ matrix.

Numerous decompositions have been proposed by means of covariance or coherence matrix. Pottier [4] classifies algorithms based on the covariance or coherence matrices for instance Cloude, Holm, Freeman, van Zyl and CloudePottier. Cloude-Pottier algorithm [5] is widely known as the Entropy-Alpha and has been employed on various applications (for example on agriculture [6] and forest monitoring [7]).

According to the Reciprocity Theorem, it is observable that $S_{h v}=S_{v h}=S_{X}$. Value of $S_{X}$ could be obtained by averaging from the measurement. Based on the assumption of three general types of scattering, the scattering matrix could be rewritten into

$$
\begin{aligned}
& {\left[\begin{array}{ll}
S_{h h} & S_{X} \\
S_{X} & S_{v v}
\end{array}\right]=\alpha\left[S_{a}\right]+\beta\left[S_{b}\right]+\gamma\left[S_{c}\right]} \\
& {\left[S_{a}\right]=\frac{1}{\sqrt{2}}\left[\begin{array}{cc}
1 & 0 \\
0 & 1
\end{array}\right] ;\left[S_{b}\right]=\frac{1}{\sqrt{2}}\left[\begin{array}{cc}
1 & 0 \\
0 & -1
\end{array}\right] ;\left[S_{c}\right]=\frac{1}{\sqrt{2}}\left[\begin{array}{ll}
0 & 1 \\
1 & 0
\end{array}\right] ;} \\
& \alpha=\frac{S_{h h}+S_{v v}}{\sqrt{2}} ; \beta=\frac{S_{h h}-S_{v v}}{\sqrt{2}} ; \gamma=S_{h v} \sqrt{2} ;
\end{aligned}
$$

$S a$ and $S b$ represent odd and even bounce scatterers respectively. Whereas $S c$ contains information on scatterers located in the 45 degrees tilted plane.

Cloude-Pottier decomposition [5] is based on the eigenvector analysis of the coherence matrix. The matrix may be given as

$$
\langle[T]\rangle=\left[U_{3}\right][\Lambda]\left[U_{3}\right]^{T}=\left[U_{3}\right]\left[\begin{array}{ccc}
\lambda_{1} & 0 & 0 \\
0 & \lambda_{2} & 0 \\
0 & 0 & \lambda_{3}
\end{array}\right]\left[U_{3}\right]^{T}
$$




$$
\left[U_{3}\right]=\left[\begin{array}{ccc}
\cos \left(\alpha_{1}\right) & \cos \left(\alpha_{2}\right) & \cos \left(\alpha_{3}\right) \\
\sin \left(\alpha_{1}\right) \cos \left(\beta_{1}\right) e^{i \delta_{1}} & \sin \left(\alpha_{2}\right) \cos \left(\beta_{2}\right) e^{i \delta 2} & \sin \left(\alpha_{3}\right) \cos \left(\beta_{3}\right) e^{i \delta 3} \\
\sin \left(\alpha_{1}\right) \cos \left(\beta_{1}\right) e^{i \gamma 1} & \sin \left(\alpha_{2}\right) \cos \left(\beta_{2}\right) e^{i \gamma 2} & \sin \left(\alpha_{3}\right) \cos \left(\beta_{3}\right) e^{i \gamma_{3}}
\end{array}\right]
$$

where $[\Lambda]$ is the diagonal eigenvalue matrix of $[\mathrm{T}], \lambda_{1}>=\lambda_{2}>=\lambda_{3}>=0$ are the real eigenvalues and the $[\mathrm{U}]$ is a unitary matrix whose columns correspond to the orthonormal eigenvectors $e_{1}, e_{2}, e_{3}$ of [T].

Coherence matrix $[T]$ can be decomposed further into

$$
[T]=\sum_{n=1}^{3} \lambda_{n}\left[T_{n}\right]=\lambda_{1}\left(e_{1} \cdot e_{1}^{T}\right)+\lambda_{2}\left(e_{2} \cdot e_{2}{ }^{T}\right)+\lambda_{3}\left(e_{3} \cdot e_{3}{ }^{T}\right)
$$

Each matrix $[T n]$ is a unitary matrix representing a deterministic scattering. The amount of contribution is given by the eigenvalues while the type of scattering is related to the eigenvectors.

There are two important components of Cloude-Pottier decomposition. First component of the decomposition is Entropy. The component is useful to measure component distribution of scattering process. Mathematically, the Entropy is defined as

$$
H=\sum_{i=1}^{3}-P_{i} \log _{3} P_{i} ; P_{i}=\frac{\lambda_{i}}{\sum_{j=1}^{3} \lambda_{j}}
$$

The definition dictates the range of Entropy of 0 to 1 . Low values indicate $[T]$ matrix has only one non-zero eigenvalue and represents one deterministic scattering process (pure targets), whereas high value means no dominant scatterer has been found (distributed targets).

The second component is alpha angle that represents scattering mechanism. With this component, separation and identification of mechanisms are being evaluated. Based on the Pauli decomposition, $[S]$ matrix could be represented by scattering vector $k$

$$
\vec{k}=\vec{k}_{P}=\left[\begin{array}{c}
S_{h h}+S_{v v} \\
S_{h h}-S_{v v} \\
2 S_{X}
\end{array}\right]
$$


Since three dimensional unitary complex vector $k$ has five degrees of freedom, it can be parameterized in terms of a set of five angles [8]

$$
\vec{k}=\left[\begin{array}{c}
\cos (\alpha) e^{i \phi} \\
\sin (\alpha) \cos (\beta) e^{i \delta} \\
\sin (\alpha) \sin (\beta) e^{i \gamma}
\end{array}\right]
$$

A change of angle $\alpha$ and $\beta$ corresponds to a differential change from one scattering mechanism $k$ to another $\left(k^{\prime}\right)$

$$
\begin{aligned}
& \vec{k}^{\prime}=\left[R_{1}\right] \vec{k}=\left[\begin{array}{ccc}
1 & 0 & 0 \\
0 & \cos (\Delta \beta) & -\sin (\Delta \beta) \\
0 & \sin (\Delta \beta) & \cos (\Delta \beta)
\end{array}\right] \cdot \vec{k} \\
& \vec{k}^{\prime}=\left[R_{2}\right] \vec{k}=\left[\begin{array}{ccc}
\cos (\Delta \alpha) & -\sin (\Delta \alpha) & 0 \\
\sin (\Delta \alpha) & \cos (\Delta \alpha) & 0 \\
0 & 0 & 1
\end{array}\right] \cdot \vec{k}
\end{aligned}
$$

Cloude and Pottier [8] developed Scattering Vector Reduction Theorem: any polarimetric backscattering mechanism, represented by a complex unitary vector $k$ and obeying reciprocity, can be reduced to the identity $\left[\begin{array}{lll}1 & 0 & 0\end{array}\right]^{T}$ by a series of 3 matrix transformations

$$
\left[\begin{array}{l}
1 \\
0 \\
0
\end{array}\right]=\left[\begin{array}{ccc}
\cos (\alpha) & \sin (\alpha) & 0 \\
-\sin (\alpha) & \cos (\Delta \alpha) & 0 \\
0 & 0 & 1
\end{array}\right]\left[\begin{array}{ccc}
1 & 0 & 0 \\
0 & \cos (\beta) & \sin (\beta) \\
0 & \sin (\beta) & \cos (\beta)
\end{array}\right]\left[\begin{array}{ccc}
e^{i \phi} & 0 & 0 \\
0 & e^{i \delta} & 0 \\
0 & 0 & e^{i \gamma}
\end{array}\right] \cdot \vec{k}
$$

Alpha angle represents target scattering type and has value ranges from 0 to 90 degrees. Values of 0,45 and 90 degrees indicate odd bounce, dipole and even bounce respectively. Orientation of the scatterers can be obtained from Beta angle, spans from -180 to 180 degrees. Another three angles are the target phase angles.

Additional feature has been presented by Pottier [9] known as Anisotropy. The feature is mathematically defined as

$$
A=\frac{\lambda_{2}-\lambda_{3}}{\lambda_{2}+\lambda_{3}}
$$

Anisotropy is highly linked with Entropy. Hence, the interpretation should be conducted in conjunction with the Entropy. 


\section{$3 \quad$ Methodology}

\subsection{Site Description and Dataset}

This experiment was situated in a coastal region of East Kalimantan (Sungai Wain region). The main data were the AirSAR fully polarimetric data acquired within PACRIM mission in 16 September 2000. The center of acquisition was located at -1.08 latitudes and 116.85 longitudes. Cross-track swath was $8.3 \mathrm{~km}$ (ground range) along $60.8 \mathrm{~km}$. Figure 1 presents the Sungai Wain test site.

The AirSAR fully polarimetric data consist of three frequencies, i.e. C- (5.3 $\mathrm{GHz}), \mathrm{L}-(1.2 \mathrm{GHz})$ and P-band $(0.45 \mathrm{GHz})$. However, in this experiment, only C-band fully polarimetric dataset was utilized to assess coastal land cover. The data were pre-processed in 30 April 2001 by the Jet Propulsion Laboratory/NASA.

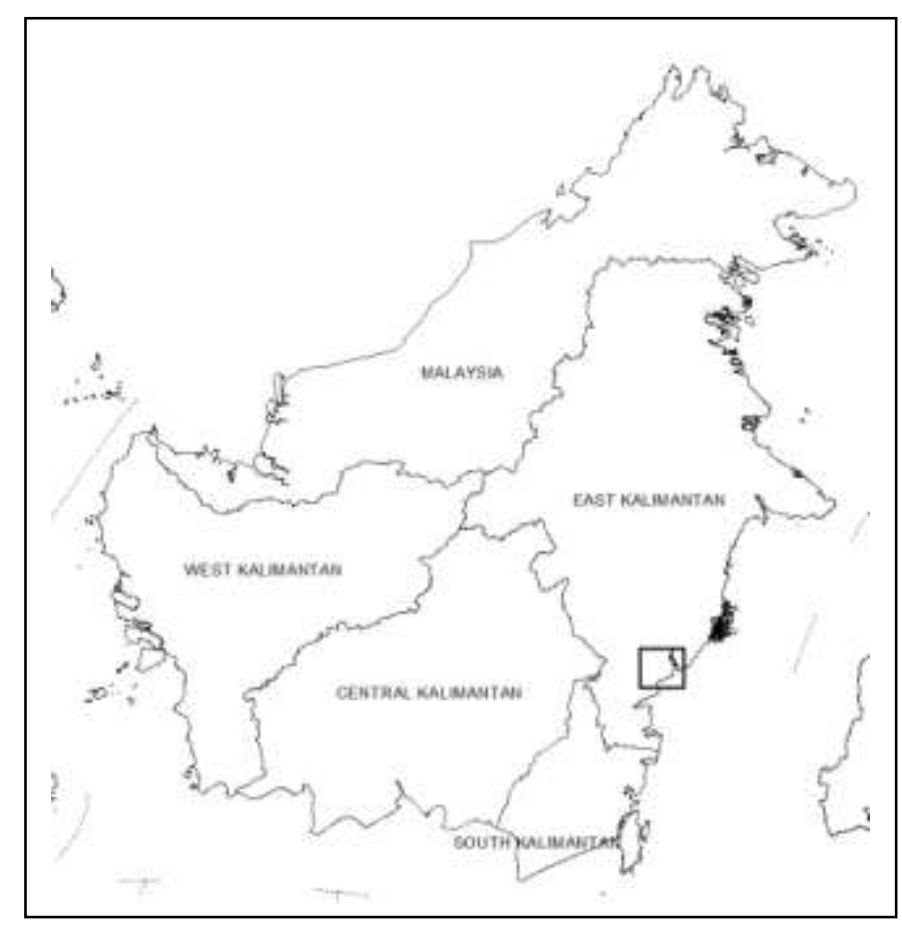

Figure 1 Site location.

\subsection{Data Analysis}

Data analysis was started by Pauli decomposition. The decomposition is useful to analyze the image visually, allowing inspection on the quality of image. The 
decomposition provides three images, which then could be used to construct a composite image. Following equation was then used to produce a composite image.

$$
R=\left|S_{h h}-S_{v v}\right| ; G=\left|S_{h v}+S_{v h}\right| ; B=\left|S_{h h}+S_{v v}\right|
$$

Another technique could be implemented to provide composite image, so called Sinclair composite. Sinclair decomposition uses Eq. 12 to construct a composite image from scattering matrix.

$$
R=\left|S_{v v}\right| ; G=\left|S_{h v}\right| ; B=\left|S_{h h}\right|
$$

AirSAR flight covered a very wide area, span from coastal to hilly regions. In order to get focused in Sungai Wain coastal area, the image was then subsetted. The region has a diverse land cover, including ponds, lowland forest, clear-cut areas and man-made structures. Subsetting was also useful to select sub area which has less defects or artifacts as previously inspected from Pauli composite. No filtering was performed in order to assess performance of proposed classification algorithms.

Classification based on the Cloude-Pottier Decomposition initially only has 9 classes, which could be segmented from Entropy-Alpha angle feature space. In order to extend the capability, a Wishart Classification algorithm proposed by Lee, et al. [10] was implemented. Initially, fully polarimetric data were segmented from Entropy-Alpha feature space. Result of the segmentation was then employed as training sets for maximum likelihood classification based on the Wishart distribution of polarimetric covariance matrix. The classified results were then exploited as training sets in order to compute a new distance measures for the next iteration [11]. In this experiment, 10 iterations were applied to allow sufficient groupings between clusters having short distance.

\section{$4 \quad$ Results and Discussion}

Before actual classification was taken, the Pauli composite image produced from Eq. (11) was carefully assessed. Since examination was taken visually, converting the absolute product of Pauli decomposition into 8-bit image was performed. The decomposition produces three important information of Shh, $S v v$ and $S x$. The odd bounce scattering component is represented by $S h h+S v v$. Components of Shh-Svv and $S x$ inform even bounce and 45 degrees tilted even bounce scattering mechanisms respectively.

Practical meaning of this configuration is that general flat surface such as sea or another water bodies are shown in blue or bluish colors. Another implication is 
that forested area will be presented in green, since major vegetation cover produces even bounce scattering mechanism. Strong backscatter from manmade features indicates dominance of even bounce scattering mechanism and colored as reddish. Result of Pauli decomposition arranged in color composite is presented in Figure 2(a).

In order to assess the performance of Cloude-Pottier decomposition, no ensemble averaging has been performed. This will lead to coarser classified image, due to the presence of texture. Readers should note that AirSAR has produced pixel spacing around 3.3 and 4.6 meters in across and along-track respectively. Hence, the texture is significant in this case.

The experiment involves two approaches mentioned in previous sections. The first is original concept of Entropy-Alpha angle presented by Cloude and Pottier [5]. The second is after insertion of additional component by Pottier [9], i.e. Anisotropy. Figure 2(b) and 2(c) present classification results after classification based on Entropy-Alpha angle and Entropy-Alpha angle-Anisotropy respectively.

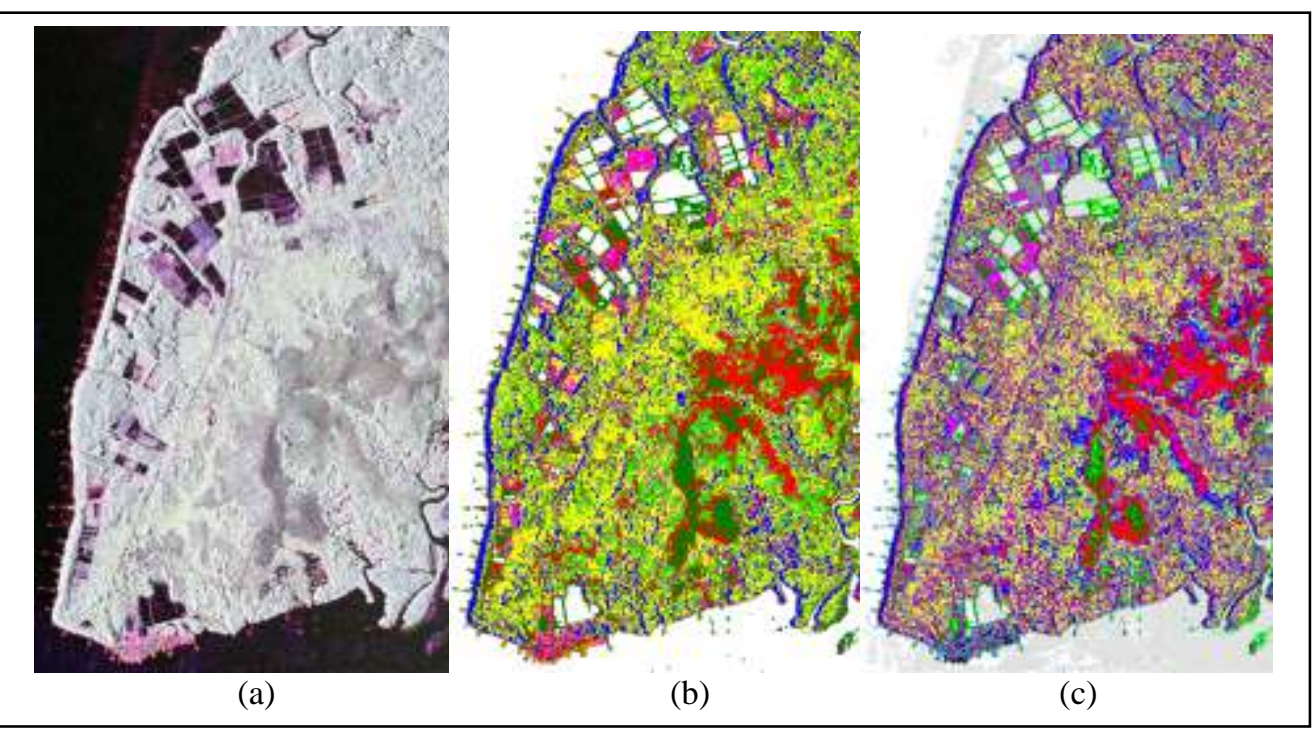

Figure 2 Pauli-based composite image (a), and corresponding classification algorithms using Entropy-Alpha (b) and Entropy-Alpha-Anisotropy (c) dataset.

Fewer classes are found in Entropy-Alpha classification. The algorithms produced 8 and 16 classes for Entropy-Alpha and Entropy-Alpha-Anisotropy respectively. Grouping of similar clusters has been indicated; hence more 
contiguous feature can be presented. In general, Entropy-Alpha classification provided contiguous classes than the Entropy-Alpha-Anisotropy. However, fewer detected classes also mean less capability to sense more features which might be available from the dataset. Following discussions are focused into several identifiable objects such as water bodies; less vegetated and/or clear-cut areas, forest and man-made structures.

Most estuaries in Kalimantan suffer from high depositions due to erosion in the uphills. In Sungai Wain site, very long and shallow deposition was identified in riverbanks. Pauli decomposition clearly shows this deposition. Due to grouping of similar clusters between water and shallow deposition in Entropy-Alpha, no separation is presented. On the other hand, Entropy-Alpha-Anisotropy provided a good discrimination between those surfaces.

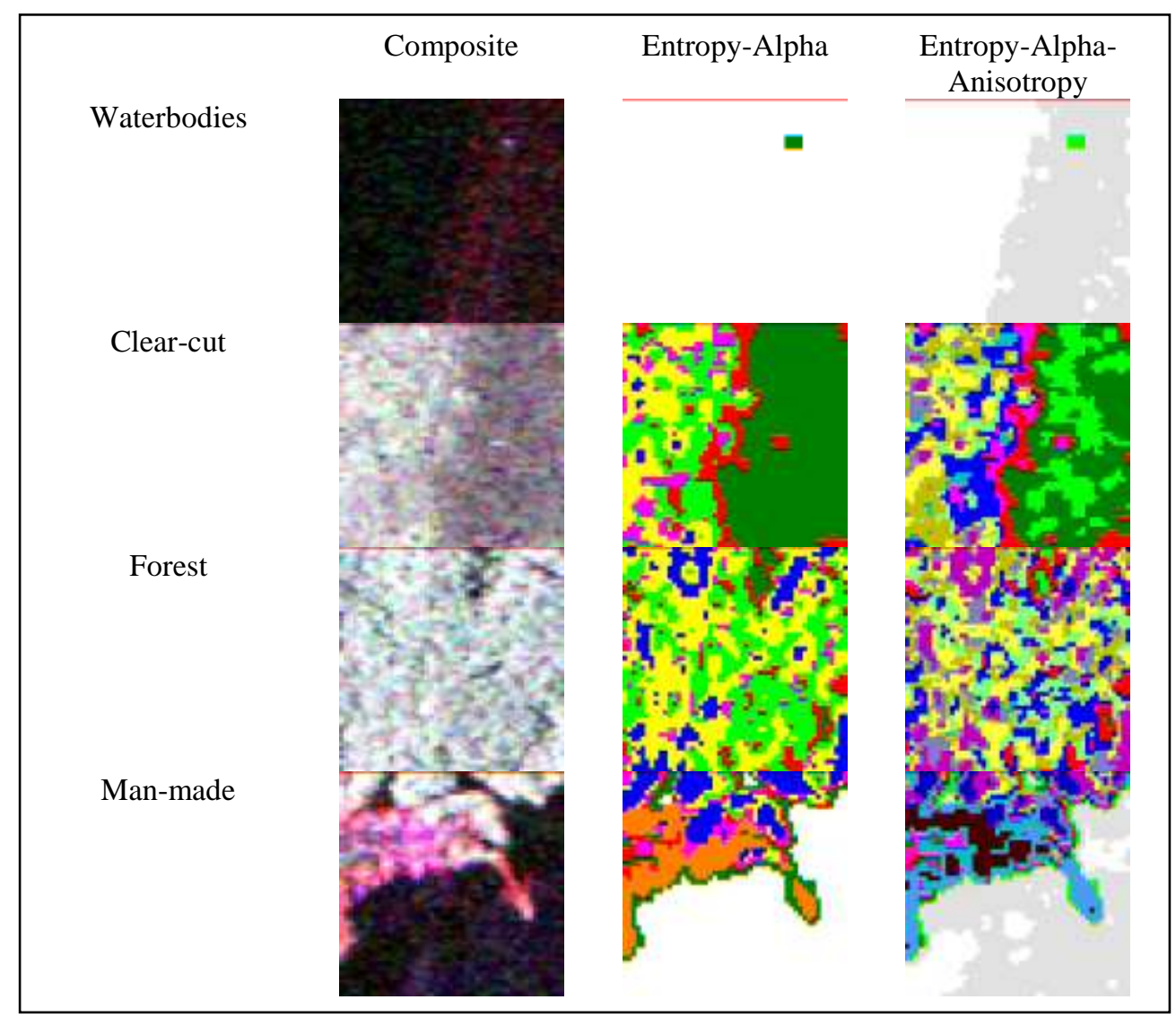

Figure 3 Detailed comparison on land uses. 
In the forest margin region, several clear-cut forests are visible in big patches. The regions are usually surrounded by agricultural fields. Results presented in Figure 3 show that classification by Entropy-Alpha provided reasonable groupings. Instead, Entropy-Alpha-Anisotropy produced an over-sensitive separation. Hence, small deviations in the clear-cut region are then separated into different clusters.

Assessment in forested area was considered the most difficult task in this experiment. The main reason is related to higher spatial resolution of AirSAR system which in turn reveals textural information. This artifact is shown in Figure 3. Both classification algorithms were unable to aggregate scatterers into single class of forest. However, it seems that the Entropy-Alpha classification provided more realistic condition. Hellmann [11] also mentioned the difficulties of $\mathrm{X}$ and $\mathrm{C}$ band for distinguishing forest and low/medium vegetation. The problem was occurred due to the limited depth of penetration. Hence, scattering mechanism of forest canopy cannot be discriminated from low and medium vegetation.

Man-made structures were easily found within this site. In SAR context, this provides major source of even bounce scattering mechanism. Pauli decomposition visualizes these structures as reddish due to insertion of even bounce scatterers into red layer. Both algorithms performed well, as the structures were recognized in specific clusters. Nonetheless, Entropy-Alpha provided more contiguous delineation than the Entropy-Alpha-Anisotropy.

\section{$5 \quad$ Conclusions}

Generally, Entropy-Alpha was found to have a higher capability to produce more contiguous area than the Entropy-Alpha-Anisotropy algorithm. For less heterogeneous areas and less number of classes being considered, preference should be made for Entropy-Alpha classification scheme. The conclusion was generally drawn at the most of sub sites. A more realistic grouping was presented by Entropy-Alpha.

In case of region which has diverse land covers, application of Entropy-Alpha may leads to less accurate results. Addition of Anisotropy may contribute significantly to detection of difficult cases. This was observable in discrimination between water and shallow depositions.

It is concluded that the Cloude-Pottier decomposition provided an efficient way to extract land cover information from surface scatterers. Within this test site, many objects have been successfully identified. However, problems persist in forested regions which may lead to further research and experiments. 


\section{Acknowledgements}

Financial support by Indonesian Ministry of Research and Technology through RUTI-II/EIRBEX is acknowledged, which initiated the interest in polarimetric data processing. Fully polarimetric multi-frequency data were provided by NASA/JPL. Sincere thanks are due to AirSAR team including Dr. B. Chapman. Dr. L. Ferro-Famil of University of Rennes-1, France deserves a special mention for his help during data processing. Careful reading by Dr. D.N. Choesin and an anonymous reviewer significantly improved the manuscript.

\section{References}

[1] Melack, J.M., Tropical Freshwater Wetlands, Manual of Remote Sensing $3^{\text {rd }}$ Ed. 4 (S. Ustin, Ed.), John Wiley and Sons, 2004.

[2] Ahmed, M.H, El Leithy, B.M., Thompson, J.R., Flower, R.J., Ramdani, M., Ayache, F. \& Hassan, S.M., Application of Remote Sensing to Site Characterisation and Environmental Change Analysis of North African Coastal Lagoons, Hydrobiologia, 622, 147-171, 2009.

[3] Huang, J., Lin, J. \& Tu, Z., Detecting Spatiotemporal Change of Land Use and Landscape Pattern in A Coastal Gulf Region, Southeast of China, Environment, Development and Sustainability, 12, 35-48, 2010.

[4] Pottier, E., SAR Polarimetry and Applications, in Proc. POLinSAR Workshop, Frascati, Italy, 2005.

[5] Cloude, S.R. \& Pottier, E., An Entropy Based Classification Scheme for Land Applications of Polarimetric SAR, IEEE Transactions on Geoscience and Remote Sensing, 35, 68-78, 1997.

[6] Putignano, C., Schiavon, G., Solimini, D. \& Trisasongko, B., Unsupervised Classification of A Central Italy Landscape by Polarimetric L-band SAR data, in Proceedings IGARSS, Seoul, South Korea, 2005.

[7] Trisasongko, B.H., Tropical Mangrove Mapping Using FullyPolarimetric Radar Data, ITB Journal of Science, 41A(2), 98-109, 2009.

[8] Cloude, S.R. \& Pottier, E., Concept of Polarisation Entropy in Optical Scattering, Optical Engineering, 34, 1599-1610, 1995.

[9] Pottier, E., Unsupervised Classification Scheme and Topography Derivation of PolSAR Data Based on the $H / A / \alpha$ polarimetric Decomposition Theorem, in Proceedings 4th International Workshop Radar Polarimetry, Nantes, France, 1998.

[10] Lee, J.-S., Grunes, M.R., Ainsworth, T.L., Du, L-J., Schuler, D.L. \& Cloude, S.R., Unsupervised Classification Using Polarimetric Decomposition and The Complex Wishart Classifier, IEEE Transactions on Geoscience and Remote Sensing, 37, 2249-2258, 1999. 
[11] Hellmann, M, Classification of Fully Polarimetric SAR-Data for Cartographic Applications, Dissertation, Technische Universität Dresden, Germany, 2000. 